\title{
The effect of temperature and gas Reynolds number on evaporation of a sessile liquid drop in mini-channel
}

\author{
Evgeniy Orlik ${ }^{1,2, a}$, Elena Bykovskaya ${ }^{1}$ and Bo-Feng Bai ${ }^{3}$ \\ ${ }^{1}$ Institute of Thermophysics, 1, Lavrentiev Ave., 630090, Novosibirsk, Russia \\ ${ }^{2}$ Novosibirsk State Agricultural University, 630039, Novosibirsk, Russia \\ ${ }^{3}$ State key lab of Multiphase Flow in Power Engineering, Xi'an Jiaotong University, 710049, Xi'an, China
}

\begin{abstract}
Experimental setup has been designed and manufactured to study the evaporation processes of liquid drop under blowing gas in mini-channel. The height of channel can be varied from 3 to $20 \mathrm{~mm}$. Substrates are removable and its surface temperature is kept to constant value. The shadow method is main measurement technique. Series of experiments with $100 \mu 1$ water drop on polished stainless still substrate are carried out in channel with $9 \mathrm{~mm}$ height. Dependences of evaporating rate for different range of temperatures and gas Reynolds numbers are obtained.
\end{abstract}

\section{Introduction}

Drop is a fairly simple physical object containing different complex phenomena. Evaporation of sessile liquid droplet is a key point of many processes such as painting, coating, humidification, the agriculture irrigation. Experimental studies show that the wettability and contact angle hysteresis have a significant effect on the drop evaporation rate $[1,2]$ and thermocapillary film rupture [3]. It is known that surfactants affect the wettability, hence there is a need that properties of test liquid be carefully characterized. Also the contact angle hysteresis has an effect on the spreading of the drop on a solid surface under gravity, which is shown in [4]. Effect of gravity on evaporation has to be considered in space applications. In recent paper [5] it is shown that Marangoni effect (flow driven by surface tension gradient) modifies the drop interface shape and impacts on evaporation. Deep understanding of evaporation requires knowledge what is happening inside droplet. Experimental works and numerical simulations show that evaporation leads to convective flow inside droplet with appearing vortex structures [6]. Such experimental investigation requires development of new optical methods, one of them based on Micro-PIV. Gas action on evaporation of drop is also known and studied [7], but mostly all investigations have been carried out in open ambient air, where conditions are not very controlled. Dynamics of drop on surface by a shearing air in channel have been studied in [8], it is shown strong influence of contact angle hysteresis and drop sizes on the dynamics. This experimental work presents results of evaporation of sessile drop on a heated substrate in mini channel under blowing air laminar flow.

\footnotetext{
${ }^{a}$ Corresponding author: orlik.evgeniy@gmail.com
} 


\section{Experimental setup}

Experimental setup with changeable height of channel from 3 to $20 \mathrm{~mm}$ is shown in Fig. 1. The goal is to study the effect of the substrate temperature and Reynolds number of air flow on the evaporation rate of a sessile drop. Distilled deionized nano-filtered water was used as the working liquid which is obtained from Milli-Q system. Initial drop volume was slightly varied and was around $100 \mu$ l. Polished stainless steel substrate was used in the experiments. The substrate temperature was maintained at 25.0, 50.0 and $70.0 \pm 0.25^{\circ} \mathrm{C}$ using Peltier elements and PR-59 controller. The gas temperature was held at $25^{\circ} \mathrm{C}$ and Reynolds number was varied from 0 to 2000 using Bronkhorst flow regulator. Flow rate in outlet was measured with Bronkhorst flow meter. Developed laminar flow was obtained due to long inlet length which is 10 times of channel height. The channel was equipped with optical glasses from top and side. The drop shape was visualized from the top and from the side with the help of the shadow technique with resolution of $6 \mu \mathrm{m} /$ pix (as shown in Fig. 2 and 3). The images from the side were processed with the Drop Shape Analysis software by KRÜSS using different methods including YoungLaplace method [9]. The advancing static contact angle was $\theta=85 \pm 6^{\circ}$.

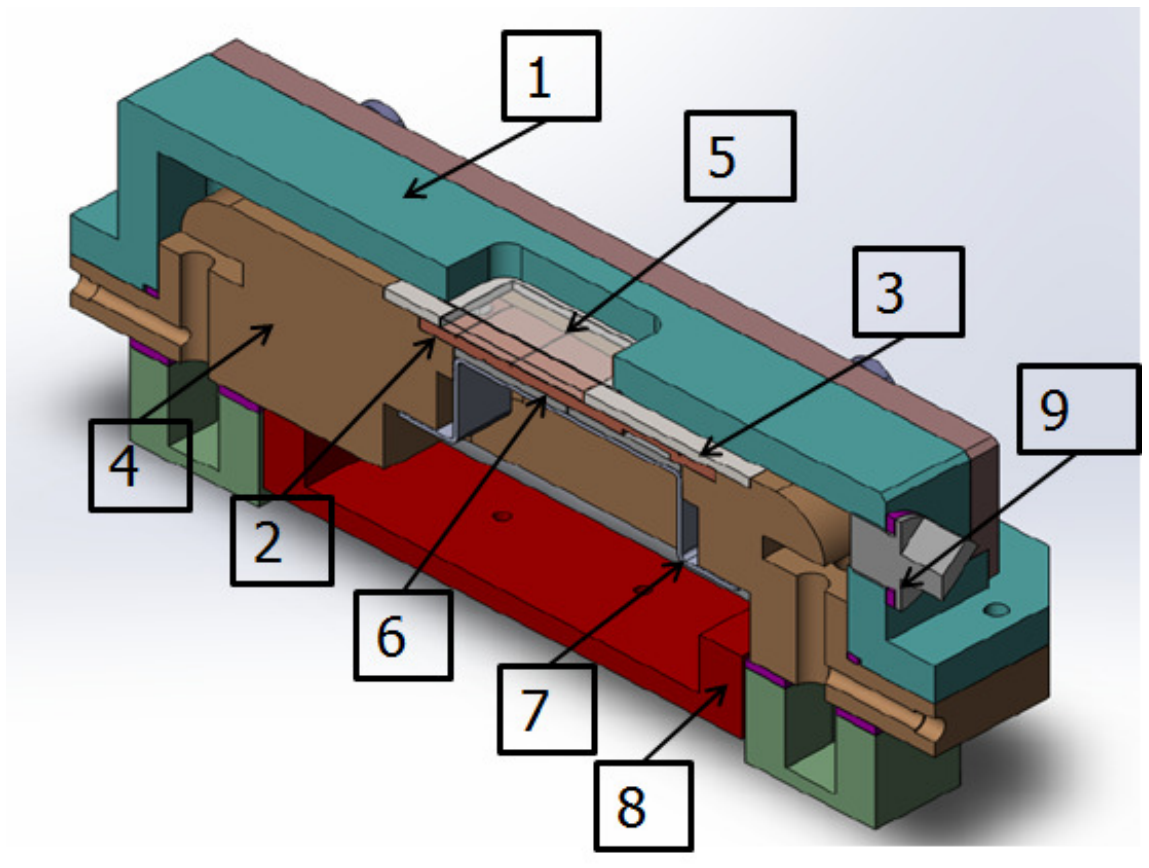

Figure 1. Experimental setup. 1 - cover, 2 - copper insert, 3 - stainless steel substrate, 5 - optical window, 6 - Peltier element, 7 - heat exchanger, 8 - support, 9 - port for syringe. 


\section{Telecentric light} source
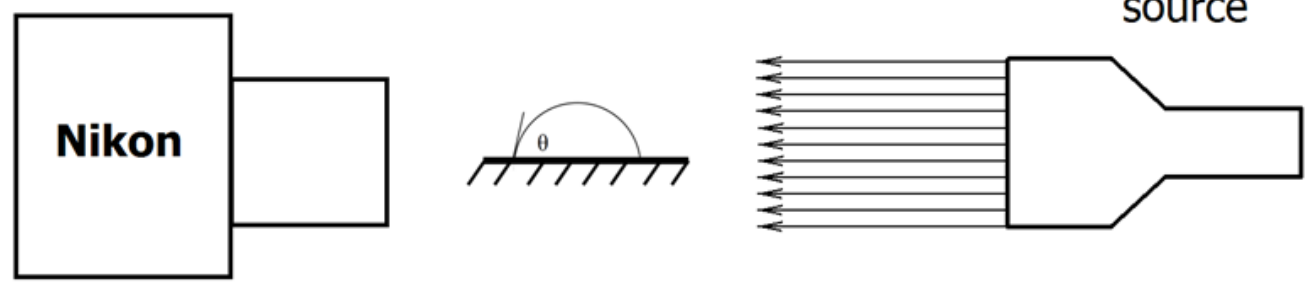

Figure 2. Schematic of the shadow technique with optical resolution of $6 \mu \mathrm{m} / \mathrm{pix}$.
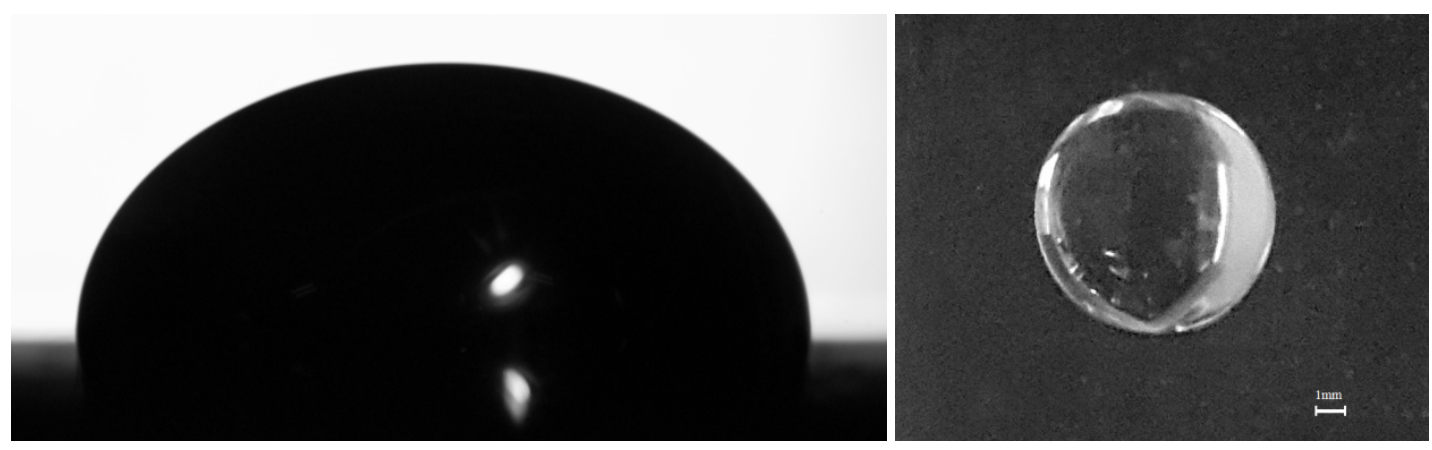

Figure 3. Photographs of $100 \mu 1$ water drop on polished stainless still substrate (side and top view).

\section{Results}

First experimental results have been obtained in channel with height of $9 \mathrm{~mm}$. The life time of liquid droplet on unheated substrate depending on Reynolds number is shown in Fig. 4. Evaporation rate which is inversely to life time is proportional to Reynolds number at power 0.285.

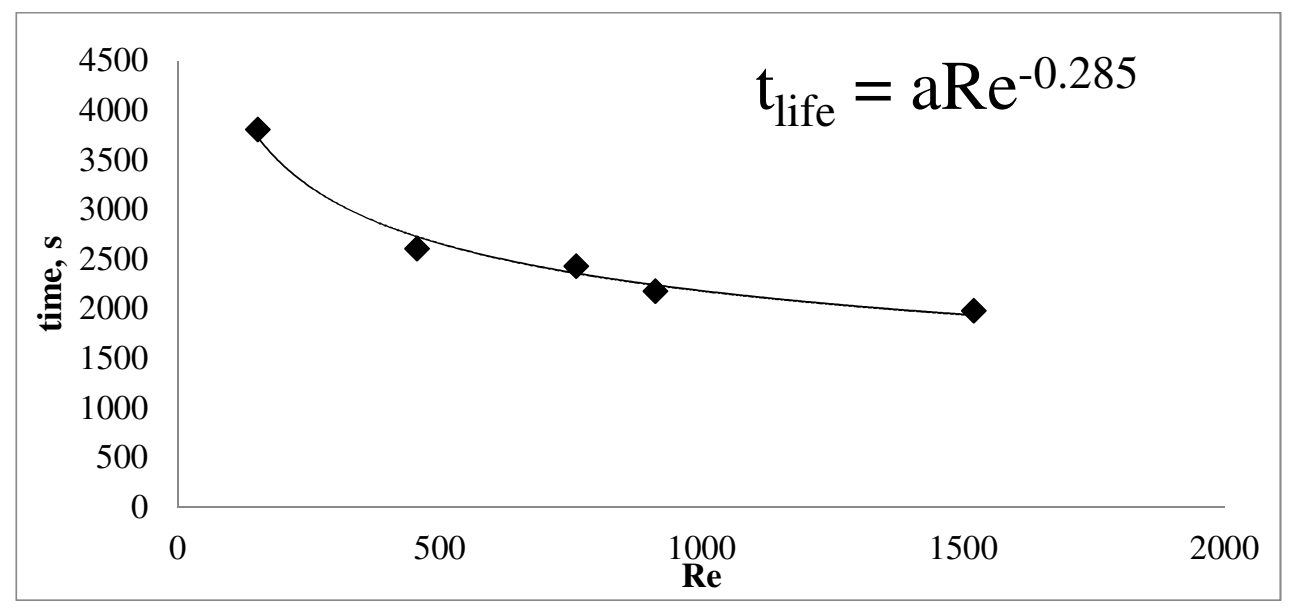

Figure4. Evaporation time of water drop vs. gas Reynolds number. Temperature of substrate is $25^{\circ} \mathrm{C}$. 
Influence of substrate temperature on evaporation rate is shown in Fig. 5. It is seen that the rate of the drop mass loss (evaporation rate) is almost constant with time. From analysis of the experimental data, we obtained that in the range of $\mathrm{Re}=1140-1900$ the evaporation rate is defined by the substrate temperature as: $\mathrm{dV} / \mathrm{dt} \approx 0.042+0.004 *\left(\mathrm{~T}_{\text {substrate }}-\mathrm{T}_{25}\right)$. Comparison of evaporation rates $\mathrm{dV} / \mathrm{dt}$ depending on substrate temperature $T$ and gas velocity $U$ for different liquids is shown in Table 1 , the data for Ethanol and HFE7100 taken from [10]. Mass evaporation rate Q in units of $\mathrm{kg} /\left(\mathrm{s} \mathrm{m}^{2}\right)$ has been measured in [10], but evaporation area in experiments [10] is constant, hence Q dV/dt. From Table 1 one can see that the data are in qualitative agreement.

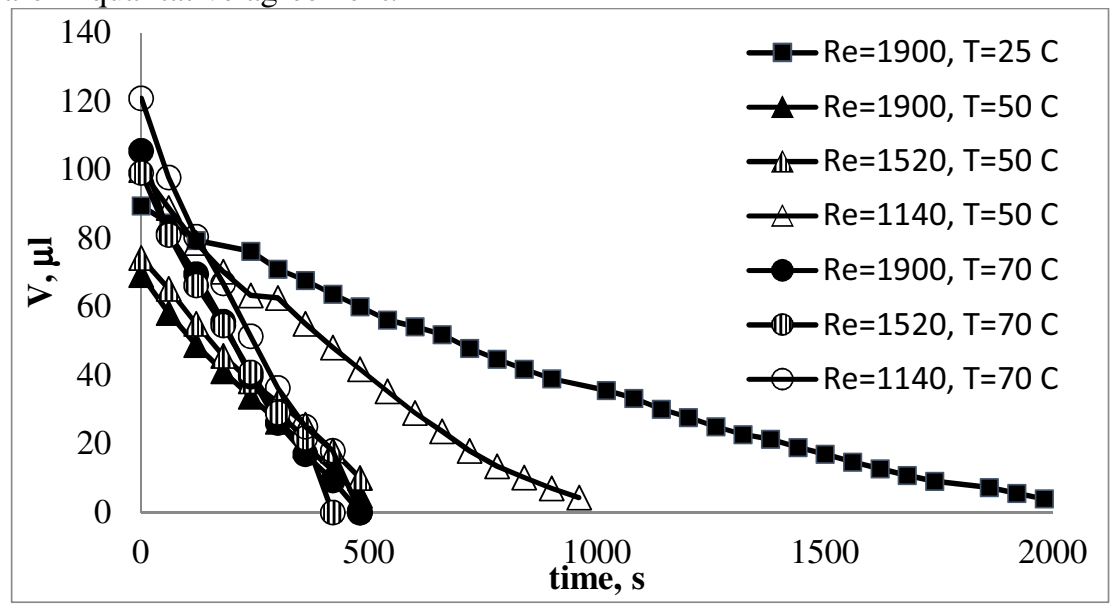

Figure 5. Change of drop volume with time for different substrate temperatures and gas Reynolds numbers.

Table 1.

\begin{tabular}{|c|c|c|c|}
\hline $\mathrm{dV} / \mathrm{dt} \sim \mathrm{T}^{\mathrm{a}}, \mathrm{dV} / \mathrm{dt} \sim \mathrm{U}^{\mathrm{b}}$ & Water sessile drop & $\begin{array}{c}\text { Liquid surface } \\
\text { (Ethanol -air) }\end{array}$ & $\begin{array}{c}\text { Liquid surface } \\
\text { (HFE7100-Nitrogen) }\end{array}$ \\
\hline $\mathrm{a}$ & 1 & 1.17 & 1.22 \\
\hline $\mathrm{b}$ & 0.285 & 0.54 & 0.176 \\
\hline
\end{tabular}

The work was financially supported by the Russian Ministry of Education and Science (Project identifier RFMEFI61315X0038).

\section{References}

1. E.Ya. Gatapova, A.A. Semenov, D.V. Zaitsev, O.A. Kabov, Colloids and Surfaces A: Physicochemical and Engineering Aspects, 441, 776, (2014)

2. A.A. Semenov, D.V. Feoktistov, D.V. Zaitsev, G.V. Kuznetsov, O.A. Kabov, Thermophysics and Aeromechanics, 22, 771, (2015)

3. D.V. Zaitsev, D.P. Kirichenko, and O.A. Kabov, Technical Physics Letters, 41, 551, (2015)

4. O.A. Kabov, D.V. Zaitsev, Doklady Physics, 58, 292, (2013)

5. Y. Tsoumpas, S. Dehaeck, A. Rednikov, P. Colinet, Langmuir, 31, 13334, (2015)

6. P.J. S'aenz, K. Sefiane, J. Kim, O.K. Matar, P. Valluri, Journal of Fluid Mechanics 772, 705-739, (2015)

7. L. Bin, R. Bennacer, A. Bouvet, Applied Thermal Engineering, 31, 3792-3798, (2011)

8. J. Fan, M.C.T. Wilson, N. Kapur, Journal of Colloid and Interface Science, 356, 286-292, (2011)

9. I.V. Marchuk, V.V. Cheverda, P.A. Strizhak, O.A. Kabov, Thermophysics and Aeromechanics 22, 297-303, (2015)

10. Yu. V. Lyulin, D. V. Feoktistov, I. A. Afanas'ev, E. S. Chachilo, O. A. Kabov, G. V. Kuznetsov, Technical Physics Letters, 41, 665-666, (2015) 